\title{
Deep inelastic scattering as a probe of entanglement: Confronting experimental data
}

\author{
Dmitri E. Kharzeev $\circledast^{1,2, *}$ and Eugene Levin $\oplus^{3,4, \uparrow}$ \\ ${ }^{1}$ Center for Nuclear Theory, Department of Physics and Astronomy, Stony Brook University, \\ New York 11794-3800, USA \\ ${ }^{2}$ Department of Physics and RIKEN-BNL Research Center, Brookhaven National Laboratory, \\ Upton, New York 11973-5000, USA \\ ${ }^{3}$ Department of Particle Physics, School of Physics and Astronomy, Tel Aviv University, \\ Tel Aviv 69978, Israel \\ ${ }^{4}$ Departamento de Física, Universidad Técnica Federico Santa María and Centro Científico-Tecnológico \\ de Valparaíso, Casilla 110-V, Valparaiso, Chile
}

(Received 22 February 2021; accepted 10 August 2021; published 30 August 2021)

\begin{abstract}
Parton distributions can be defined in terms of the entropy of entanglement between the spatial region probed by deep inelastic scattering and the rest of the proton. For very small $x$, the proton becomes a maximally entangled state. This approach leads to a simple relation $S=\ln N$ between the average number $N$ of color-singlet dipoles in the proton wave function and the entropy of the produced hadronic state $S$. At small $x$, the multiplicity of dipoles is given by the gluon structure function, $N=x G\left(x, Q^{2}\right)$. Recently, the H1 collaboration analyzed the entropy of the produced hadronic state in deep inelastic scattering, and studied its relation to the gluon structure function; poor agreement with the predicted relation was found. In this paper we argue that a more accurate account of the number of color-singlet dipoles in the kinematics of $\mathrm{H} 1$ experiment (where hadrons are detected in the current fragmentation region) is given not by $x G\left(x, Q^{2}\right)$ but by the sea quark structure function $x \Sigma\left(x, Q^{2}\right)$. Sea quarks originate from the splitting of gluons, so at small $x x \Sigma\left(x, Q^{2}\right) \sim x G\left(x, Q^{2}\right)$, but in the current fragmentation region this proportionality is distorted by the contribution of the quark-antiquark pair produced by the virtual photon splitting. In addition, the multiplicity of color-singlet dipoles in the current fragmentation region is quite small, and one needs to include $\sim 1 / N$ corrections to $S=\ln N$ asymptotic formula. Taking both of these modifications into account, we find that the data from the $\mathrm{H} 1$ collaboration in fact agree well with the prediction based on entanglement.
\end{abstract}

DOI: 10.1103/PhysRevD.104.L031503

In our paper [1] (see also [2,3]) we computed the von Neumann entropy of the system of partons resolved by deep inelastic scattering (DIS) at a given Bjorken $x$ and momentum transfer $q^{2}=-Q^{2}$. We then proposed to interpret it as the entropy of entanglement between the spatial region probed by deep inelastic scattering and the rest of the proton. We found that in the small $x$, large rapidity $Y$ regime, all partonic microstates have equal probabilities - the proton is composed by an exponentially large number $N$ of microstates that occur with equal and small probabilities $1 / N$. This yields a simple

\footnotetext{
*Dmitri.Kharzeev@stonybrook.edu

†leving@tauex.tau.ac.il,eugeny.levin@usm.cl
}

Published by the American Physical Society under the terms of the Creative Commons Attribution 4.0 International license. Further distribution of this work must maintain attribution to the author(s) and the published article's title, journal citation, and DOI. Funded by SCOAP ${ }^{3}$. relation between the entanglement entropy and the multiplicity of partons (dominated by gluons at small $x$ ):

$$
S=\ln [N]
$$

where $N\left(x, Q^{2}\right)$ is an average number of color-singlet dipoles. In the region of small $x$ the gluons dominate and $N \simeq x G\left(x, Q^{2}\right)$ where $x G\left(x, Q^{2}\right)$ is the gluon structure function. ${ }^{\prime}$ Assuming that the multiplicity of produced hadrons is proportional to the multiplicity of color-singlet dipoles ("local parton-hadron duality" [4,5]), Eq. (1) imposes a relation between the parton structure function (extracted from the inclusive cross section of DIS) and the entropy of produced hadrons; this relation can be directly tested in experiment. The comparison to the experimental

\footnotetext{
${ }^{1}$ Note that this relation is a quantum analog of the Boltzmann formula underlying statistical physics.
} 
data on hadron multiplicity distributions from CMS collaboration at the LHC provided encouraging results [1,2].

However, recent dedicated experimental analysis performed by the $\mathrm{H} 1$ collaboration [6] shows a disagreement with Eq. (1) if one assumes $N=x G\left(x, Q^{2}\right)$ (see Fig. 12 in Ref. [6] and the dotted curves in our Fig. 1). In this paper we demonstrate that the $\mathrm{H} 1$ data in fact are in a good agreement with our approach, once two important effects are taken into account. Both of them are implied by the kinematics of the $\mathrm{H} 1$ measurements that are performed in the current fragmentation region (see also Refs. [6-9]), and thus at moderate values of Bjorken $x$. First, since the experimental hadron multiplicities are not large, we need to take into account corrections of the order of $1 / N$ to Eq. (1). Second, because the $\mathrm{H} 1$ data are concentrated in the current fragmentation region and not at very small $x$, we need to reconsider our claim in Ref. [1] that the multiplicity of the color-singlet dipoles is equal to $x G\left(x, Q^{2}\right)$. Indeed, the hadrons produced in the current fragmentation region of DIS originate from the hadronization of the struck quark (a constituent of the color-singlet dipole) and the multiplicity of color-singlet dipoles is thus determined by the sea quark structure function, see Fig. 2. Therefore the correct relation between the number of dipoles and the experimentally measured entropy of hadrons (valid for large $N$ ) is

$$
S_{\text {dipoles }}=\ln \left(x \Sigma\left(x, Q^{2}\right)\right)=S_{\text {hadron }} .
$$

The sea quark and gluon distributions are related to each other at small $x$ by (see Fig. 2)

$x \Sigma\left(x, Q^{2}\right)=C\left(\alpha_{s} \ln Q^{2}, \alpha_{s}, \alpha_{s} \ln (1 / x)\right) x G\left(x, Q^{2}\right)$,

where the function $C\left(\alpha_{s} \ln Q^{2}, \alpha_{s}, \alpha_{s} \ln (1 / x)\right)$ describes the splitting of the virtual photon into the quark-antiquark pair. In the region of very small $x$, as we will now show, $C\left(\ln Q^{2}, \alpha_{s}, \alpha_{s} \ln (1 / x)\right) \rightarrow$ Const, and so the sea quark distribution is proportional to the gluon one. Therefore, with a logarithmic accuracy at very small $x$ we recover our original relation

$$
S_{\text {hadron }}=\ln \left(x G\left(x, Q^{2}\right)\right) .
$$

To show that $C\left(\ln Q^{2}, \alpha_{s}, \alpha_{s} \ln (1 / x)\right) \rightarrow$ Const at very small $x$, let us use the leading order Dokshitzer-Gribov-LipatovAltarelli-Parisi evolution equation [10] that gives for the sea quark structure function (see Fig. 2)
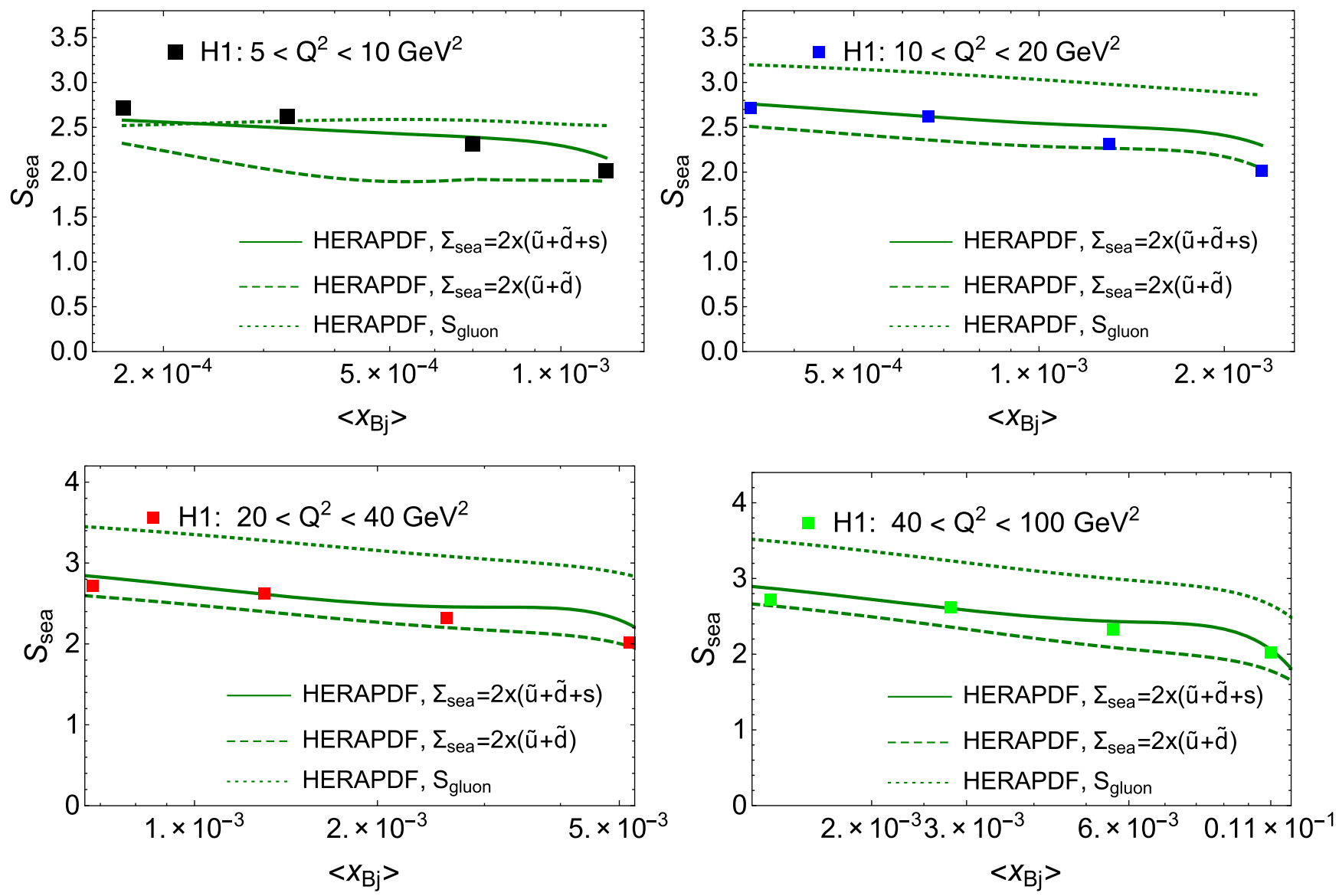

FIG. 1. Comparison of the experimental data of the H1 collaboration [6] on the entropy of produced hadrons in DIS [6] with our theoretical predictions, for which we use the sea quark distributions from the NNLO fit [12,13] to the combined H1-ZEUS data. 


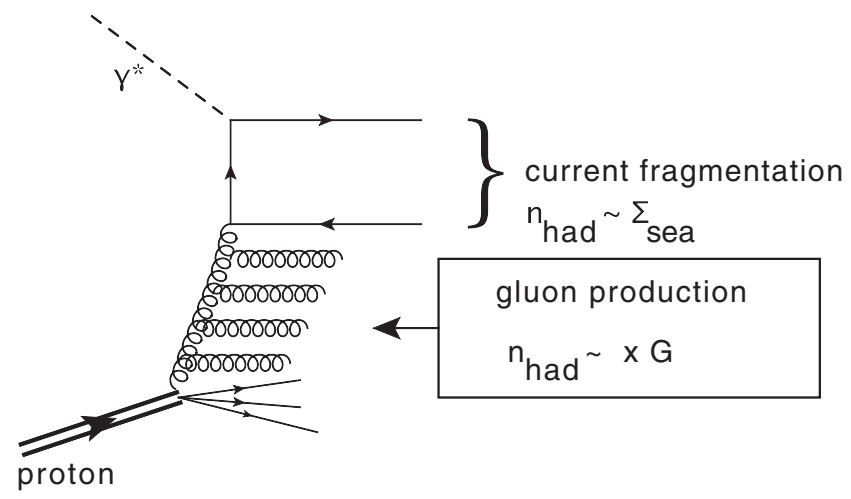

FIG. 2. DIS at small $x$.

$$
x \Sigma\left(x, Q^{2}\right)=\frac{C_{F} \alpha_{s}}{2 \pi} \int_{0}^{\xi} d \xi^{\prime} \int_{x}^{1} d z P_{q G}(z)\left(\frac{x}{z} G\left(\frac{x}{z}, \xi^{\prime}\right)\right)
$$

with $P_{q G}(z)=\frac{1+(1-z)^{2}}{z}$,

where $\xi=\ln Q^{2}$. At small values of $x$, the gluon structure function takes the form (see Ref. [11] for a review)

$$
x G(x, \xi)=\int_{\epsilon-i \infty}^{\epsilon+i \infty} \frac{d \gamma}{2 \pi i}\left(\frac{1}{x}\right)^{\alpha_{s} \frac{N_{c}}{\pi} \chi(\gamma)} e^{\gamma \xi} g(\gamma)_{\text {in }},
$$

where $N_{c}$ is the number of colors, $C_{F}=\left(N_{c}^{2}-1\right) / 2 N_{c}$, and the Balitsky-Fadin-Kuraev-Lipatov kernel $\chi(\gamma)$ has the form:

$$
\begin{aligned}
\chi(\gamma) & =2 \psi(1)-\psi(\gamma)-\psi(1-\gamma) \\
& \stackrel{\gamma \rightarrow \frac{1}{2}}{\rightarrow} \underbrace{4 \ln 2}_{\omega_{0}}+\underbrace{14 \zeta(3)}_{D}\left(\gamma-\frac{1}{2}\right)^{2} \text { in diffusion approximation; }
\end{aligned}
$$

$g(\gamma)_{\text {in }}$ should be calculated from the initial conditions. Plugging Eq. (6) in Eq. (5) we can take the integrals over $z$ and $\xi^{\prime}$. Taking the integral over $\gamma$ in the diffusion approximation, and using the method of steepest descent, we obtain the following result:

$$
x \Sigma\left(x, Q^{2}\right)=\frac{2 C_{F}}{\omega_{0} N_{c}} x G\left(x, Q^{2}\right)=\text { Const } x G\left(x, Q^{2}\right) .
$$

We evaluated Eq. (8) for small $\alpha_{s}$ neglecting terms in $C\left(\alpha_{s} \ln Q^{2}, \alpha_{s}, \alpha_{s} \ln (1 / x)\right)$ proportional to $x$. Note that Const does not depend on $\alpha_{s}$ and numerically is about 0.3 . Actually, the next-to-leading order correction increases the value of this Const and, as we can see from Fig. 1 (panel with $5 \leq Q^{2} \leq 10 \mathrm{GeV}^{2}$ ), Const $\sim 1$ in the next-to-next-toleading order (NNLO) fit for the region of smallest $x$. However, away from the region of very small $x$ and in the kinematics of $\mathrm{H} 1$ measurement, the proportionality (8) is violated, and one should use the relation (2) instead of (4).
In addition, away from small $x$, where the multiplicity of color-singlet dipoles $N$ is not large, one should take into account $1 / N$ corrections to (4). In Refs. [1,3] it is shown that in QCD cascade the multiplicity distribution has the following form:

$$
p_{n}(N)=\frac{1}{N}\left(1-\frac{1}{N}\right)^{n-1}
$$

where $N$ is the average multiplicity of color-singlet dipoles. The distribution (9) leads to the following von Neumann entropy:

$S=-\sum p_{n} \ln p_{n}=\ln (N-1)+N \ln \left(1+\frac{1}{N-1}\right)$.

One can see that at large $N$ we obtain $S \simeq \ln N$, but corrections are sizable when $N \leq 10$ (see Fig. 3). It should be noted that the distribution of Eq. (9) describes quite well the experimental hadron multiplicity distributions in proton-proton collisions (see Refs. [1-3]).

For comparison with the H1 experimental data [6] (see Fig. 1), we first assume, following [1], that the hadron multiplicity is equal to the number of color-singlet dipoles. This assumption is based on "parton liberation" picture [5] and on the "local parton-hadron duality" [4]. For sea quark and gluon structure functions in Fig. 1 we use NNLO fit $[12,13]$ to the combined H1 and ZEUS data.

One can see that our approach in fact describes the $\mathrm{H} 1$ data quite well - this is the first test of the relation between entanglement and the parton model in DIS enabled by the $\mathrm{H} 1$ analysis. We stress that once the data in the target fragmentation region at smaller value of $x$ becomes available at the electron-ion collider, one should be able to use $x G\left(x, Q^{2}\right)$ in the relation (1), as it has been done in Refs. $[1-3,14,15]$. However, the general formula is given by Eq. (2) which at small $x$ reduces to $S=\ln \left(x G\left(x, Q^{2}\right)\right)$ since $x \Sigma\left(x, Q^{2}\right) \rightarrow x G\left(x, Q^{2}\right)$.

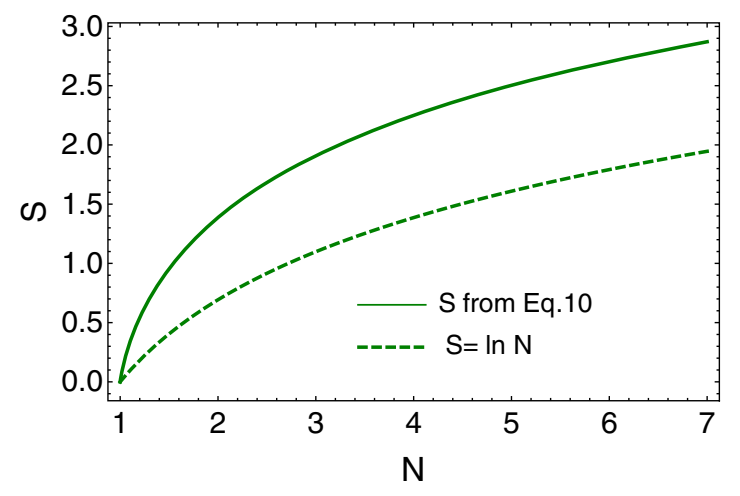

FIG. 3. Entropy versus multiplicity $N$ from Eqs. (1) and (10). 
We thank Kong Tu, Thomas Ullrich, and our colleagues at BNL, Stony Brook University, Tel Aviv University and UTFSM for stimulating discussions. We are very grateful to Aharon Levy and Katarzyna Wichmann for help in finding and extracting the parton distributions in the NNLO fit to HERA data. This work was supported in part by the U.S. Department of Energy under Contracts No. DE-FG88ER40388 and No. DESC0012704, BSF Grant No. 2012124, Chilean National Agency for Research and Development PIA/APOYO AFB180002 (Chile) and Fondecyt (Chile) Grant No. 1180118.
[1] D. E. Kharzeev and E. M. Levin, Deep inelastic scattering as a probe of entanglement, Phys. Rev. D 95, 114008 (2017).

[2] Z. Tu, D. E. Kharzeev, and T. Ullrich, Einstein-PodolskyRosen Paradox and Quantum Entanglement at Subnucleonic Scales, Phys. Rev. Lett. 124, 062001 (2020).

[3] E. Gotsman and E. Levin, High energy QCD: Multiplicity distribution and entanglement entropy, Phys. Rev. D 102, 074008 (2020).

[4] Y. L. Dokshitzer, V. A. Khoze, S. I. Troian, and A. H. Mueller, QCD coherence in high-energy reactions, Rev. Mod. Phys. 60, 373 (1988).

[5] A. H. Mueller, Toward equilibration in the early stages after a high-energy heavy ion collision, Nucl. Phys. B572, 227 (2000).

[6] V. Andreev et al. (H1 Collaboration), Measurement of charged particle multiplicity distributions in DIS at HERA and its implication to entanglement entropy of partons, Eur. Phys. J. C 81, 212 (2021).

[7] C. Adloff et al. (H1 Collaboration), Multiplicity structure of the hadronic final state in diffractive deep inelastic scattering at HERA, Eur. Phys. J. C 5, 439 (1998).

[8] C. Adloff et al. (H1 Collaboration), Evolution of e $\mathrm{p}$ fragmentation and multiplicity distributions in the Breit frame, Nucl. Phys. B504, 3 (1997).

[9] S. Chekanov et al. (ZEUS Collaboration), Energy dependence of the charged multiplicity in deep inelastic scattering at HERA, J. High Energy Phys. 06 (2008) 061.
[10] V. N. Gribov and L. N. Lipatov, Deep inelastic e p scattering in perturbation theory, Yad. Fiz. 15, 781 (1972) [Sov. J. Nucl. Phys. 15, 438 (1972)]; G. Altarelli and G. Parisi, Asymptotic freedom in parton language, Nucl. Phys. B126, 298 (1977); Y. L. Dokshitzer, Calculation of the structure functions for deep inelastic scattering and $e+e-$ annihilation by perturbation theory in quantum chromodynamics., Zh. Eksp. Teor. Fiz. 73, 1216 (1977) [Sov. Phys. JETP 46, 641 (1977)].

[11] Y. V. Kovchegov and E. Levin, Quantum Choromodynamics at High Energies, Cambridge Monographs on Particle Physics, Nuclear Physics and Cosmology (Cambridge University Press, Cambridge, England, 2012).

[12] H. Abramowicz et al. (H1 and ZEUS Collaborations), Combination of measurements of inclusive deep inelastic $e^{ \pm} p$ scattering cross sections and QCD analysis of HERA data, Eur. Phys. J. C 75, 580 (2015).

[13] K. Wichmann (private communication).

[14] O. K. Baker and D. E. Kharzeev, Thermal radiation and entanglement in proton-proton collisions at energies available at the CERN large hadron collider, Phys. Rev. D 98, 054007 (2018).

[15] E. Gotsman and E. Levin, Thermal radiation and inclusive production in the CGC/saturation approach at high energies, Eur. Phys. J. C 79, 415 (2019). 